\title{
FRECUENCIA DE VIRUS DEL PAPILOMA HUMANO EN ATIPIA DE CÉLULAS ESCAMOSAS DE SIGNIFICADO INDETERMINADO
} SERIE DE CASOS

Carlos H. Pérez MD*, Miryam P. de Amaya**, Astrid C. López MD***, Adriana Delgado MD****, Silvia M. Plata MD****

\section{Resumen}

El cáncer de cuello uterino resulta de una infección persistente por el virus del papiloma humano (VPH). Las políticas mundiales de salud se basan en programas de tamizaje para disminuir la incidencia del cáncer. Se han implementado pruebas para detectar el VPH y así esclarecer informes citomorfológicos ambiguos como la atipia de células escamosas de significado indeterminado (ASC-US), para optimizar el manejo de estos resultados citológicos. Objetivos: establecer la frecuencia de VPH de alto riesgo mediante prueba molecular en mujeres con diagnóstico de ASC-US por citología convencional en el Hospital de San José de Bogotá DC. Métodos: serie de casos con citología convencional, colposcopia y prueba para VPH, con seguimiento a seis meses. Resultados: de 121 pacientes $16 \%$ tuvieron diagnóstico de ASC-US y en 15\% de estas la prueba de VPH de alto riesgo fue positiva. Conclusiones: la frecuencia de VPH en casos con ASCUS fue de 15\%, comparable con lo reportado en la literatura, y en la totalidad de la muestra el VPH estuvo en 11.5\%. De los factores de riesgo no se encontró relación con inicio temprano de relaciones sexuales y el número de compañeros sexuales.

Palabras clave: cytology, DNA probes, HPV, human papillomavirus, cervix uteri, uterine cervical neoplasms.

Abreviaturas: VPH, virus del papiloma humano; ASC-US, atipia de células escamosas de significado indeterminado: $\mathrm{BR}$, bajo riesgo; $\mathrm{AR}$; alto riesgo.

\section{FREQUENCY OF HUMAN PAPILLOMA VIRUS IN WOMEN DIAGNOSED WITH ATYPICAL SQUA MOUS CELLS OF UNDETERMINED SIGNIFICANCE}

\section{Abstract}

Uterine cervix cancer results from a persistent infection by the human papilloma virus (HPV). World heath policies are based on screening programs to reduce cancer incidence. HPV testing has been implemented to clarify ambivalent

Fecha recibido: mayo 29 de 2014 - Fecha aceptado: junio 24 de 2014

* Médico ginecólogo. Colposcopista, ProfesorTitular. Fundación Universitaria de Ciencias de la Salud, Bogotá DC, Colombia.

** Bacterióloga y Citohistóloga. Docente de patólogos y citólogos desde 1967. Profesora Asistente, Fundación Universitaria de Ciencias de la Salud, Bogotá DC, Colombia.
*** Residente IV de Ginecología y Obstetricia. Fundación Universitaria de Ciencias de la Salud, Bogotá DC, Colombia.

**** Residente III de Ginecología y Obstetricia. Fundación Universitaria de Ciencias de la Salud. Bogotá DC, Colombia. 
cytology morphologic reports such as atypical squamous cells of undetermined significance (ASCUS), to optimize the management of these cytology results. Objectives: to establish the frequency of high-risk HPV using a molecular probe in women with an ASCUS diagnosis obtained by conventional cytology at Hospital de San José de Bogotá DC. Methods: case series performing a conventional cytology, colposcopy HPV test and a six-month follow-up. Results: of 121 patients $16 \%$ had an ASCUS diagnosis and a positive high-risk HPV test in $15 \%$ of these cases. Conclusions: the frequency of positive HPV tests in women with an ASCUS diagnosis was $15 \%$, comparable with that reported in literature. HPV test was positive in $\mathbf{1 1 . 5 \%}$ of the total sample. No relation between sexual activity at an early age and multiple sexual partners was found among the risk factors.

Key words: cytology, DNA probes, HPV, human papillomavirus, cervix uterine neoplasms

\section{Introduc ción}

El cáncer de cuello uterino es el resultado de una infección persistente por el virus papiloma humano (VPH) ${ }^{1}$, tiene una incidencia de 40 por 100.000 mujeres al año en países en desarrollo y es una de las causas más frecuentes de muerte en mujeres en el mundo. ${ }^{2}$ Mientras América del sur y el suroccidente africano tienen una incidencia mayor de 20 por cada 100.000 mujeres por año, los países desarrollados de Europa occidental, América del Norte, Nueva Zelanda y Australia tienen una de 10 por $100.000 .{ }^{3}$ Colombia tiene una incidencia de cáncer de cuello uterino de 12.6/100.000 mujeres al año. ${ }^{4}$

Las políticas de salud a nivel mundial han estado enfocadas a desarrollar programas de tamizaje con citología convencional para disminuir la incidencia de cáncer de cuello uterino, con una detección precoz de las lesiones en el cérvix. ${ }^{5}$ En los países desarrollados, cuando lograron un adecuado cubrimiento de los programas de tamizaje, se modificó la incidencia y la mortalidad asociada con el cáncer de cuello uterino, por la detección temprana de lesiones del cérvix. ${ }^{6-8}$

En Colombia, durante más de 40 años los programas de tamizaje de cáncer de cérvix han tenido como herramienta la citología cérvicovaginal convencional. Los programas organizados de tamizaje en países desarrollados han disminuido la mortalidad hasta en $80 \%$. La reducción se explica por estrategias como educación de la población, búsqueda activa de pacientes, adecuados sistemas de información y entrega de resultados, diagnóstico y tratamiento oportunos. ${ }^{9,10}$ En los países en vía de desarrollo como el nuestro, los programas de tamización no han tenido el mismo impacto en la mortalidad. ${ }^{11}$

El sistema de clasificación para la citología cérvicovaginal Bethesda 2001 incluye una categoría llamada "atipias en células escamosas de significado indeterminado" (ASC-US). En esta los criterios citomorfológicos no son cualitativa o cuantitativamente suficientes para denominarse como lesión intraepitelial de bajo (LIE BG) o de alto grado. Existen otros factores que parecen estar relacionados con el resultado de ASC-US en la citología como son los cambios reactivos o reparativos/ regenerativos, así como la presencia de otros microorganismos que forman parte de la flora patógena. ${ }^{12}$

El 99,8\% de los cánceres de cérvix se asocian con infección persistente por tipos específicos de VPH. Algunos serotipos se relacionan con el desarrollo de carcinomas anogenitales (cérvix, vagina, vulva, ano, pene), por lo que son denominados como VPH de alto riesgo (VPH AR), en esta categoría se incluyen los serotipos 16, 18, 31, 33, 35, 39, 45, 51, 52, 56, 58, 59, 68,73 . Cerca del $70 \%$ de los cánceres se asocian con infección por VPH 16 y $18 .{ }^{13}$ Las técnicas basadas en la detección de antígenos o del ADN del VPH, 
mediante hibridación o amplificación ${ }^{14}$ permiten esclarecer resultados ambiguos como ASC-US. ${ }^{15}$ El uso de pruebas para la detección de VPH están aprobadas en países desarrollados como Estados Unidos, Reino Unido y Canadá. La Food and Drug Administration (FDA) autorizó las pruebas de VPH como método de detección en 1996. ${ }^{16}$ En Colombia esto ocurrió a través del acuerdo 029 de 2011 y las aprobó el INVIMA (Instituto Nacional de Vigilancia de Medicamentos y Alimentos) para su uso en el plan obligatorio de salud. La prueba de PCR (del inglés polymerase chain reaction) para VPH (COBAS 4800) autorizada por la FDA, es específica para los VPH-AR 16 y 18, además de otros 12 serotipos asociados también con el desarrollo de cáncer de cérvix. La prueba Cobas 4800 tiene validación clínica para ASC-US y al identificar VPH-AR permite enfocar el manejo de las pacientes ya que una carga viral elevada es un factor de riesgo para el desarrollo de carcinoma in-situ y puede predecir su aparición antes de las alteraciones en la citología cérvicovaginal. ${ }^{14}$ Dada la importancia de la infección por VPH en el desarrollo de las lesiones cervicales, se hace necesario determinar cuál es la frecuencia de la infección por VPH de alto riesgo en la población colombiana y así tener en cuenta los factores de riesgo para realizar intervenciones oportunas y lograr disminuir la incidencia del tumor.

\section{Metodología}

Estudio descriptivo de tipo serie de casos, que incluyó mujeres que asistieron en forma voluntaria a las campañas de promoción y prevención de cáncer de cuello uterino programadas por las facultades de citohistología y medicina de la Fundación Universitaria de Ciencias de la Salud (FUCS) durante 2011-2012. Se realizó citología convencional, colposcopia y prueba de PCR para VPH (Cobas 4800). Se excluyeron las pacientes embarazadas, célibes o que hubieran recibido tratamiento oncológico previo (radio o quimioterapia).

Las citologías cervicovaginales y las colposcopias fueron realizadas por el personal del servicio de colposcopia del Hospital de San José de Bogotá DC, y las primeras se analizaron en la facultad de citohistología de la FUCS, interpretadas según los criterios Bethesda
2001. Los casos positivos (definidos como ASC-US o mayor) y dudosos fueron revisados y firmados por un patólogo, así como el 10\% de los casos negativos cumpliendo con el control de calidad interno.

A las mujeres incluidas en el estudio se practicó prueba de VPH Cobas 4800 (Roche Molecular Diagnostics, Pleasanton, CA, USA) que cuenta con preparación automatizada, combinada con la tecnología de PCR en tiempo real para la amplificación y detección simultánea de 14 genotipos del VPH de alto riesgo y la identificación específica de VPH 16 y 18. La $\beta$-globina fue utilizada como control interno para la validez de la muestra. Las pruebas de PCR fueron procesadas en el laboratorio ROCHE. ${ }^{17}$

En la recolección de los datos se revisaron reportes citocolposcópicos de las mujeres que asistieron a las campañas de promoción y prevención realizadas por la FUCS (campaña $\mathrm{N}^{\circ}$ 1: 29 de septiembre de 2011; campaña $\mathrm{N}^{\circ}$ 2: 25 de junio de 2012). Los datos se consignaron en el instrumento de recolección incluyendo variables sociodemográficas como edad, número de compañeros sexuales e inicio de relaciones sexuales, así como los resultados de la citología convencional y en base liquida, colposcopia, biopsia (se tomó a quienes tuvieron colposcopia positiva para cambios mayores o menores) y resultados de pruebas de VPH reportados como negativos o positivos para VPH 16,18 o para otros virus de alto riesgo. A las pacientes con ASCUS se les practicó colposcopia y prueba de VPH, con control de citología y colposcopia a los seis meses.

Las variables cualitativas se resumieron en frecuencias absolutas y relativas, las cuantitativas con medidas de tendencia central y de dispersión. El análisis estadístico se realizó en Stata versión 12. El protocolo fue aprobado por el comité de ética en investigación con seres humanos de la facultad de medicina de la FUCS.

\section{Resultados}

Se incluyeron 121 pacientes que asistieron a las campañas de promoción y prevención ya mencionadas. La edad mediana fue 37,5 y se incluyeron tanto mujeres en edad fértil como postmenopáusicas. La 
mayoría eran multíparas y se encontró igual proporción de nulíparas y primíparas $(24.1 \%)$. Se obtuvo información del número de compañeros sexuales de 110 pacientes, de las cuales $65.7 \%$ referían dos o menos (Tabla 1).

\section{VPH y número de compañeros sexuales}

De las mujeres que tuvieron dos o menos compañeros sexuales $11.1 \%$ fueron positivas para VPH AR, $1.38 \%$ para los serotipos 16 o 18. Con más de tres compañeros en $15.7 \%$ el resultado fue positivo para VPH de alto riesgo, $2.63 \%$ de ellas para serotipos 16 o 18 (Tabla 2).

\begin{tabular}{|c|c|}
\hline Características & $n(\%)$ \\
\hline Edad mediana ( RIQ) & $37.5(19-72)$ \\
\hline \multicolumn{2}{|l|}{ Antecedentes } \\
\hline tabaquismo & $12(10.4)$ \\
\hline \multicolumn{2}{|l|}{ Inicio temprano de RS } \\
\hline menor a 17 años & $34(3 \mathrm{I})$ \\
\hline mayor o igual a 18 años & $75(68.8)$ \\
\hline \multicolumn{2}{|l|}{ Numero de compañeros } \\
\hline menor o igual a 2 & $72(65.4)$ \\
\hline mayor a 3 & $38(34.54)$ \\
\hline \multicolumn{2}{|l|}{ Paridad } \\
\hline nulipara & $29(24.1)$ \\
\hline primípara & $29(24.1)$ \\
\hline multípara & $62(51.6)$ \\
\hline \multicolumn{2}{|l|}{ Planificación } \\
\hline no planifica & $3 I(28.1)$ \\
\hline DIU & $13(10.74)$ \\
\hline implante & $2(1.65)$ \\
\hline inyectable & II (9.09) \\
\hline ligadura & $33(27.2)$ \\
\hline píldora & $8(6.6 I)$ \\
\hline preservativo & $18(14.88)$ \\
\hline vasectomía & $2(1.65)$ \\
\hline
\end{tabular}

\section{VPH y edad de inicio de relaciones sexuales}

109 mujeres informaron la edad de inicio de relaciones sexuales, la mayoría a los 18 años o más; $14.7 \%$ de las que iniciaron antes de los 18 años fueron positivas para VPH-AR. El 10\% de las que lo hicieron después de 18 fueron positivas para VPH-AR (Tabla 3).

\section{VPH y población total}

Del total de 121 pacientes, $10.7 \%$ fueron positivas para otros serotipos de alto riesgo mientras que sólo $1,65 \%$ lo fue para VPH-16 o 18 . Ninguna presentó coinfección por VPH-16 y VPH-18 (Tabla 4); 24\%

\begin{tabular}{|l|c|c|c|}
\hline \multicolumn{4}{|c|}{ Tabla 2. Numero de compañeros } \\
sexuales y VPH \\
\begin{tabular}{|} 
No. de compañeros \\
sexuales
\end{tabular} & VPH - H & VPH I6 & VPH I8 \\
\hline menor o igual a 2 & 8 & I & I \\
\hline mayor a 3 & 4 & I & I \\
\hline Total & 12 & 2 & 2 \\
\hline
\end{tabular}

\begin{tabular}{|c|c|c|c|}
\hline $\begin{array}{l}\text { Inicio de relaciones } \\
\text { sexuales }\end{array}$ & VPH - H & VPH 16 & VPH 18 \\
\hline menor a 17 años & 5 & I & 0 \\
\hline mayor o igual 18 años & 7 & I & 2 \\
\hline Total & 12 & 2 & 2 \\
\hline
\end{tabular}

\begin{tabular}{|c|c|c|c|c|c|c|}
\hline $\begin{array}{l}\text { Cito } \\
\text { conven }\end{array}$ & $\begin{array}{l}\text { logía } \\
\text { ncional }\end{array}$ & Negativa & HPV-HR & HPV-I6 & HPV-I 8 & $\begin{array}{l}\text { Invali- } \\
\text { dada }\end{array}$ \\
\hline Negativa & $92(0.76)$ & $80(0.66)$ & $5(0.04 I)$ & I $(0.0082)$ & 0 & $8(0.066)$ \\
\hline ASCUS & $20(0.16)$ & $14(0.1 \mathrm{I})$ & $3(0.024)$ & 0 & I (0.0082) & $3(0.02)$ \\
\hline LIE-BG & $8(0.06)$ & I $(0.0082)$ & $5(0.04 I)$ & $I(0.0082)$ & $I(0.0082)$ & 0 \\
\hline Total & 121 & 95 & 13 & 2 & 2 & II \\
\hline
\end{tabular}


tuvieron citologías anormales, 7.5\% LEI-BG y las demás ASC-US.

\section{VPH y ASC-US}

El $16.5 \%$ de las citologías realizadas correspondieron a ASC-US; de ellas el 15\% cursaron con prueba positiva para VPH-AR; 5\% tuvieron coinfección por VPH-AR y VPH-18 (Tabla 4).

\section{VPH y colposcopia}

El $15.3 \%$ de las colposcopias anómalas fueron positivas para VPH, 7.7\% VPH-AR, 7.7\% VPH-16 y ninguna para VPH 18. Las pacientes con colposcopias negativas presentaron menos resultados positivos para VPH (Tabla 5).

\section{ASC-US y seguimiento}

En las mujeres con ASC-US a las cuales se les realizó biopsia por presentar colposcopia positiva, la mayoría de los reportes correspondieron a LIE-BG. En el control a los seis meses solo $0.5 \%$ de ASC-US persistieron, ninguna presentó progresión y todas las colposcopias fueron negativas (Tabla 6).

\section{Discusión}

El cáncer de cuello uterino es causado por una infección persistente por VPH-AR y es en la actualidad el cáncer ginecológico más frecuente en los países en desarrollo, por lo cual es considerado un problema de salud pública en Colombia. El VPH está formado por un genoma de doble cadena de ADN y se han caracterizado más de 120 tipos, de los cuales cerca de 40 subtipos son capaces de generar infección en el tracto genital. Alrededor de 15 de estos se clasifican como HPV-AR dadas sus propiedades oncogénicas. ${ }^{18,19}$

El VPH estuvo presente en $11.5 \%$ de las mujeres de 19 a 72 años incluidas en el presente estudio. La edad de presentación más frecuente fue en mayores de 30 años $(57.1 \%)$. En estudios a gran escala para detectar el VPH en el momento de las pruebas de citología, como el ARTISTIC, que incluyó 24.510 mujeres, el 27,3\% de las menores de 30 tenían VPH de alto riesgo, mientras que sólo el 6,1\% de las mayores de 30 años fueron positivas para VPH de alto riesgo en el momento de la citología. ${ }^{20}$ En el estudio se encontró una menor frecuencia de infección por VPH que lo reportado en la literatura mundial. Se han identificado como factores de riesgo para VPH la edad temprana de la primera relación sexual y el número de compañeros sexuales.

\begin{tabular}{|l|c|c|c|c|c|c|}
\multicolumn{7}{c}{ Tabla 5. Frecuencia de VPH en colposcopias } \\
\multicolumn{2}{c}{ Colposcopia } & Negativa & HPV-H & HPV-16 & HPV-18 & Invalidada \\
\hline Negativa & $108(0.89)$ & $83(0.68)$ & $11(0.09)$ & $1(0.0082)$ & $2(0.016)$ & $10(0.082)$ \\
\hline Positiva & $13(0.107)$ & $10(0.082)$ & $1(0.0082)$ & $1(0.0082)$ & 0 & $1(0.008)$ \\
\hline Total & 121 & 93 & 12 & 2 & 2 & 11 \\
\hline
\end{tabular}

\begin{tabular}{|c|c|c|c|c|}
\hline ASC-US & Positiva & Negativa & invalidada & total \\
\hline Colposcopia & 4 & 16 & 0 & 20 \\
\hline Biopsia & 3 & 1 & 0 & 4 \\
\hline Control Colposcopia & 0 & 4 & 0 & 4 \\
\hline Control Citología & 1 & 19 & 0 & 20 \\
\hline $\mathrm{VPH}-\mathrm{H}$ & 3 & 14 & 3 & 20 \\
\hline VPH -16 & 0 & 17 & 3 & 20 \\
\hline VPH -18 & 1 & 16 & 3 & 20 \\
\hline
\end{tabular}


La infección por VPH en adolescentes se produce con mayor frecuencia por la susceptibilidad a la entrada y persistencia viral debida a la inmadurez estructural de la zona de transformación. ${ }^{21}$

En nuestro estudio las que tuvieron dos o menos compañeros sexuales $(11.1 \%)$ fueron positivas para VPH-AR, mientras que $15.7 \%$ con más de tres compañeros tuvieron infección por VPH. Dichos datos se relacionan con lo reportado en la literatura mundial, sin embargo se debe tener en cuenta que no todas las pacientes incluidas en el estudio revelaron datos de su historia sexual.

Respecto a la edad de inicio de las relaciones sexuales, se identificó un mayor número de infección por VPH en quienes lo hicieron antes de los 18 años (14.7\% vs $10 \%$ en las que iniciaron vida sexual a los 18 años o más). Al igual que lo reportado antes para el número de compañeros sexuales, no todas dieron información de la edad de inicio de vida sexual, por lo cual tampoco es posible asignarlo como un factor de riesgo. Algunas de las razones por las cuales no brindaron toda la información o que pudo ser errónea, es porque pertenecen a la institución que va a observarlas y seguirlas.

El ASC-US es un resultado frecuente pero tiene bajo riesgo de desarrollar neoplasia intraepitelial cervical 3 ya que cerca de $75 \%$ no están relacionadas con la infección por VPH. ${ }^{22,23}$ Aquellas con ASC-US y pruebas positivas de VPH-AR tienen doble riesgo de desarrollar lesiones de alto grado. ${ }^{24-26}$ Alrededor de 10 a $20 \%$ de las mujeres con ASC-US puede presentar NIC 2 o 3 subyacente y una de cada 1.000 puede tener cáncer. ${ }^{26}$ Este aumento en el riesgo de quienes tienen VPH positivo y ASC-US en la citología, es una de las razones por las que es importante conocer la infección por VPH antes de definir el manejo.

La frecuencia de ASC-US encontrada en el presente estudio fue del $16.5 \%$, lo que se considera mayor a lo reportado en la literatura mundial. El estudio ATHENA que incluyó 47.208 mujeres con diagnóstico citológico de ASC-US reveló una prevalencia del $4.1 \% .{ }^{27} \mathrm{Sin}$ embargo, es importante tener en cuenta que fue realizado en Estados Unidos un país con menor incidencia de patología cervical que el nuestro. El realizado en Nigeria mostró ASC-US en el $42.9 \%$ de las citologías anormales. $^{27}$

- Los reportes de citología anormal en el presente estudio solo correspondían a ASC-US y LIE-BG, que puede explicarse porque habían tenido citologías previas y es posible que las lesiones cervicales estuvieran identificadas. La incidencia de ASC-US en un estudio realizado en Myanmar, entre 2010 y 2011 que incluyó 1.771 casos, 762 (43,0\%) tenían citología normal y $4,9 \%$ con ASC-US. Se identificó HPV-AR en $60 \%$ de las pacientes con ASCUS seleccionadas para la realización de la prueba mediante PCR, ADN de VPH-AR. Aquellas con citología anormal eran diez veces más propensas a ser VPH-AR positivas que con citología normal $(\mathrm{p}=0,0001){ }^{28}$

- En el estudio ATHENA se encontraron prevalencias de HVP-AR en 32.6\%, HPV-16 de $8.2 \%$ y HPV-18 de 2.9\%; en las pacientes con ASC-US y diagnóstico colposcópico de NIC 1, NIC 2 y NIC 3 se presentó en $10.1 \%, 2.2 \%$ y $2.9 \%$ respectivamente. ${ }^{13}$ En el presente estudio, $15 \%$ fueron positivas para VPH-AR y una de ellas fue positiva también para VPH-18. No se encontró VPH-16 en casos positivos para ASC-US.

- De las pacientes con ASC-US, 20\% presentaron colposcopias satisfactorias de las cuales $15 \%$ tuvieron confirmación por biopsia de LEI-BG, lo que se correlaciona con los hallazgos del estudio ATHENA. No se encontraron diagnósticos de LEI de alto grado. En el seguimiento a seis meses todas las colposcopias fueron negativas, lo cual demuestra el menor riesgo de progresión de las citologías con ASC-US cuando no están relacionadas con infección por VPH.

Las limitaciones del presente estudio están dadas por la población base del mismo, ya que al ser trabajadoras de un hospital tienen mayor acceso a los servicios de salud, por lo que las pruebas de tamizaje y la detección de lesiones cervicales se harían con mayor rigor que la población general de nuestro país, lo que limita la 
posibilidad de generalizar los resultados a la población colombiana, limitando la validez externa del estudio. La infección por el virus de papiloma humano continúa siendo un problema de salud en Colombia, por lo cual deben realizarse estudios con un mayor número de pacientes para poder generalizar los datos a la población colombiana.

\section{Conclusión}

La frecuencia de infección por virus de papiloma humano en las mujeres con diagnóstico de ASC-US, en el Hospital de San José fue 15\%, siendo menor a lo reportado en la literatura mundial.

Declaración de conflicto de interés: el investigador principal ha sido conferencista de Laboratorios ROCHE.

\section{Referencias}

1. Castellsaguè X, Llongueras $\mathrm{S}$, Bosch FX. Epidemiología de la infección por VPH y del cáncer de cuello de útero. Nuevas opciones preventivas. En: Carreras Collado R, Xercavins Montosa J, Checa Vizcaíno MA. Virus del papiloma humano y cáncer de cuello de útero. Buenos Aires: Editorial Médica Panamericana; 2007.

2. Franco EL, Duarte-Franco E, Ferenczy A. Cervical cancer: epidemiology, prevention and the role of human papillomavirus infection. CMAJ. 2001;164(7):1017-25

3. Ferlay J, Shin HR, Bray F, Forman D, Mathers C, Parkin DM. Estimates of worldwide burden of cancer in 2008: GLOBOCAN 2008. Int J Cancer 2010;127(12):2893-917.

4. WHO, International Angency for Research On Cancer. Globocan 2012. Estimated incidence, mortality and prevalence worldwide in 2012 [monograph on the Internet]. Lyon, France: IARC, World Health Organitation; 2012 [cited 2014 may 20]. Disponible en: http://globocan.iarc.fr/Pages/fact_sheets_cancer.aspx.

5. Borré Arrieta $\mathrm{O}$, Redondo C. Patología premaligna y maligna de cérvix [monografía en Internet]. Cartagena, Colombia: Clínica de Maternidad Rafael Calvo; 2012 [citado 2014 mayo 20]. Disponible en: http://www.maternidadrafaelcalvo. gov.co/protocolos/PROTOCOLO_PATOLOGIA_PREMALIGNA_Y_MALIGNA_DE_CE.pdf.

6. Macgregor JE, Campbell MK, Mann EM, Swanson KY. Screening for cervical intraepithelial neoplasia in north east Scotland shows fall in incidence and mortality from invasive cancer with concomitant rise in preinvasive disease. BMJ. 1994;308(6941):1407-11.

7. McKinley E. Principios generales de citología. En: Atkinson BF, editor. Atlas de diagnóstico citopatológico. Madrid: Elsevier; 2011. p. 1-13.

8. Tamayo R. Prólogo. En: Alonso de Ruiz P, Lazcano Ponce EC, Hernández Ávila M, editores. Cáncer cervicouterino: diagnóstico, prevención y control. Buenos Aires: Editorial Médica Panamericana; 2005.

9. Quinn M, Babb P, Jones J, Allen E. Effect of screening on incidence of and mortality from cancer of cervix in England: evaluation based on routinely collected statistics. BMJ. 1999;318(7188):904-8.
10. Peto J, Gilham C, Fletcher O, Matthews FE. The cervical cancer epidemic that screening has prevented in the UK. Lancet. 2004; 364(9430):249-56.

11. Parkin DM, Bray F, Ferlay J, Pisani P. Global cancer statistics, 2002. CA Cancer J Clin. 2005;55(2):74-108.

12. Sherman ME, Abdul-Karim F. Células escamosas atípicas. En: Solomon D, Nayar R, editores. El Sistema Bethesda para informar la citología cervical : definiciones, criterios y notas aclaratorias. Buenos Aires: Journal; 2005.

13. Doorbar J. The papillomavirus life cycle. J Clin Virol. 2005;32 Suppl 1:S7-15.

14. Stoler MH, Wright TC Jr, Sharma A, Apple R, Gutekunst K, Wright TL, et al. High-risk human papillomavirus testing in women with ASC-US cytology: results from the ATHENA HPV study. Am J Clin Pathol. 2011;135(3):468-75.

15. Dahl MB, Ejersbo D, Holund B. [Cause and follow-up of inadequate cervical smears in the county of Funen]. Ugeskr Laeger. 2002;164(37):4280-3.

16. Colgan TJ. Programmatic assessments of the clinical effectiveness of gynecologic liquid-based cytology. Cancer. 2003;99(5):259-62.

17. Castle PE, Sadorra M, Lau T, Aldrich C, Garcia FA, Kornegay J. Evaluation of a prototype real-time PCR assay for carcinogenic human papillomavirus (HPV) detection and simultaneous HPV genotype 16 (HPV16) and HPV18 genotyping. J Clin Microbiol. 2009;47(10):3344-7.

18. Castellsague X, Bosch FX, Munoz N, Meijer CJ, Shah KV, de Sanjose S, et al. Male circumcision, penile human papillomavirus infection, and cervical cancer in female partners. N Engl J Med. 2002;346(15):1105-12.

19. Schiffman M, Herrero R, Desalle R, Hildesheim A, Wacholder S, Rodriguez AC, et al. The carcinogenicity of human papillomavirus types reflects viral evolution. Virology. 2005;337(1):76-84.

20. Sargent A, Bailey A, Almonte M, Turner A, Thomson C, Peto J, et al. Prevalence of type-specific HPV infection by age and grade of cervical cytology: data from the ARTISTIC trial. Br J Cancer. 2008;98(10):1704-9.

21. Roset Bahmanyar E, Paavonen J, Naud P, Salmeron J, Chow SN, Apter D, et al. Prevalence and risk factors for cervical HPV infection and abnormalities in young adult women at enrolment in the multinational PATRICIA trial. Gynecol Oncol. 2012;127(3):440-50.

22. ASCUS-LSIL Traige Study (ALTS) Group. Results of a randomized trial on the management of cytology interpretations of atypical squamous cells of undetermined significance. Am J Obstet Gynecol. 2003;188(6):1383-92.

23. Katki HA, Schiffman M, Castle PE, Fetterman B, Poitras NE, Lorey T, et al. Five-year risks of CIN 3+ and cervical cancer among women with HPV testing of ASC-US Pap results. J Low Genit Tract Dis. 2013;17(5 Suppl 1):S36-42.

24. Einstein MH, Martens MG, Garcia FA, Ferris DG, Mitchell AL, Day SP, et al. Clinical validation of the Cervista HPV HR and 16/18 genotyping tests for use in women with ASC-US cytology. Gynecol Oncol. 2010;118(2):116-22.

25. Gage JC, Schiffman M, Solomon D, Wheeler CM, Castle PE. Comparison of measurements of human papillomavirus persistence for postcolposcopic surveillance for cervical precancerous lesions. Cancer Epidemiol Biomarkers Prev. 2010;19(7):1668-74

26. Solomon D, Schiffman M, Tarone R. Comparison of three management strategies for patients with atypical squamous cells of undetermined significance: baseline results from a randomized trial. J Natl Cancer Inst. 2001;93(4):293-9.

27. Pimentel VM, Jiang X, Mandavilli S, Umenyi Nwana C, Schnatz PF. Prevalence of high-risk cervical human papillomavirus and squamous intraepithelial lesion in Nigeria. J Low Genit Tract Dis. 2013;17(2):203-9.

28. Mu-Mu-Shwe, Harano T, Okada S, Aye-Aye-Win, Khin-Saw-Aye, Hlaing-Myat-Thu, et al. Prevalence of high-risk human papillomavirus (HR-HPV) infection among women with normal and abnormal cervical cytology in Myanmar. Acta Med Okayama. 2014;68(2):79-87. 